\title{
Energy transfers and locality in magnetohydrodynamic turbulence
}

\author{
Mahendra K. Verma, ${ }^{1}$ Arvind Ayyer, ${ }^{2}$ and Amar V. Chandra ${ }^{1}$ \\ ${ }^{1}$ Department of Physics, Indian Institute of Technology, Kanpur - 208016, INDIA \\ ${ }^{2}$ Department of Physics and Astronomy, Rutgers University, Piscataway, NJ, USA
}

(Dated: March 7, 2005)

\begin{abstract}
The shell-to-shell energy transfer rates for magnetohydrodynamic (MHD) turbulence are computed analytically, which shows local energy transfer rates from velocity to velocity, velocity to magnetic, magnetic to velocity, and magnetic to magnetic fields for nonhelical MHD in the inertial range. It is also found that for kinetic-energy dominated MHD fluid, there is a preferential shell-toshell energy transfer from kinetic to magnetic energy; the transfer is reversed for magnetic-energy dominated MHD fluid. This property is the reason for the asymptotic value of Alfvén ratio to be close to 0.5. The analytical results are in close agreement with recent numerical results. When magnetic and kinetic helicities are turned on, the helical contributions are opposite to the corresponding nonhelical contributions. The helical energy transfers have significant nonlocal components.
\end{abstract}

PACS numbers: 47.65.+a, 52.30.Cv, 52.35.Ra

\section{INTRODUCTION}

Turbulent fluid and plasma flows exhibit complex behaviour. One such phenomena is the energy transfers among various scales. For fluid turbulence the energy transfer issues have been investigated in great details. However, detailed analysis of these processes is lacking in magnetohydrodynamic (MHD) turbulence. Detailed understanding of energy transfer is useful for understanding various physical process, for example, dynamo mechanism to generate magnetic field in astrophysical objects. These results are also useful in modelling MHD flows and in simulations. For example, we need to model backscatter and forward energy transfer for large-eddy simulations. In the present paper we investigate the above issues analytically.

Kolmogorov's fluid turbulence phenomenology for incompressible turbulence is based on local energy transfer between wavenumber shells. There are several quantitative theories in fluid turbulence about the amount of energy transfer between neighbouring wavenumber shells. For examples, Kraichnan [1] showed that 35\% of the energy flux comes from wavenumber triads where the smallest wave-number is greater than one-half of the middle wavenumber. This phenomenology has been verified using numerical and analytical methods [2, 3, 4, 5]. Debliquy et al. 6] recently studied the issues of energy transfers in decaying magnetohydrodynamic (MHD) turbulence using direct numerical simulation (DNS). Alexakis et al. 7] and Mininni et al. [8] performed the similar calculations for forced MHD turbulence for both helical and nonhelical flows. They found that typically, the shell-to-shell energy transfer is local. In the present paper we compute the above quantities analytically, and compare them with the numerical results.

The interactions in MHD are through $(\mathbf{u}(\mathbf{k}), \mathbf{u}(\mathbf{p}), \mathbf{u}(\mathbf{k}-\mathbf{p}))$ and $(\mathbf{b}(\mathbf{k}), \mathbf{u}(\mathbf{p}), \mathbf{b}(\mathbf{k}-\mathbf{p}))$ triads, where $(\mathbf{u}, \mathbf{b})$ are the velocity and magnetic fields respectively, and $\mathbf{k}, \mathbf{p}$, and $\mathbf{k}-\mathbf{p}$ are the wavenumbers of the triad. Kraichnan [1] gave a general formalism to compute the magnitudes of triad interactions using transfer function $S(k \mid p, q)[9]$. In this paper we will compute the shell-to-shell energy transfer using a modified method called mode-to-mode energy transfer rate $S(k|p| q)$, which represents the energy transfer mode from $\mathbf{p}$ to mode $\mathbf{k}$, with mode $\mathbf{q}$ acting as a mediator. The new formalism is necessary for computing the shell-to-shell energy transfer because the earlier formalism suffers from ambiguity arising due to the third leg of the interaction (see [10, 11]). The calculation is done using perturbative field-theory up to first-order in perturbation. We take Kolmogorov's spectrum for the energy spectrum as discussed in current numerical and analytical papers [12, 13, 14, 15, 16, 17. Note that the field-theoretic calculations has a lot of similarity with Eddy-damped quasi-normal Markovian (EDQNM) approximation.

MHD turbulence involves interactions among velocity and magnetic modes, hence energy transfer takes place between velocity to velocity, magnetic to magnetic, velocity to magnetic, and magnetic to velocity modes. Debliquy et al. 6] computed the shell-to-shell energy transfers in decaying MHD turbulence using simulation data on $512^{3}$. In their calculation cross helicity, magnetic helicity, and kinetic helicity are negligible. They also took the mean magnetic field to zero. Debliquy et al. found forward and local energy transfer from velocity to velocity, and magnetic to magnetic fields. Regarding the velocity-to-magnetic energy transfer, for the Alfvén ratio greater than approximately 0.4, the energy transfer is from kinetic to magnetic; the transfer direction is reversed for Alfvén ratio less than 0.4. Dar et al. [10], Alexakis et al. 7] and Mininni et al. [8] have done the similar analysis for 2D and 3D forced MHD turbulence, with forcing at small wavenumber of velocity. They find local energy transfer for velocity to velocity fields, and magnetic to magnetic fields in the inertial range. However, the small-wavenumber velocity shells provide energy to the small-wavenumber magnetic shells, as well as to the inertial range magnetic shells. We will show in this 
paper that our theoretical results on inertial range energy transfers are in general agreement with the above numerical results.

Pouquet et al. [18] were the first to investigate whether interactions in MHD turbulence are local or not. Their analysis is based on eddy-damped quasi-normal Markovian (EDQNM) calculation. They claimed that nonlocal interactions exist in MHD due to the mean magnetic field (Alfvén effect) and helicity. According to Pouquet et al., the local interactions cause the energy cascade, but the nonlocal ones lead to an equipartition of kinetic and magnetic energy. In the present paper we will also show that helicity induces nonlocal energy transfers.

A detailed picture of energy transfers is very useful for understanding turbulence and its modelling. In this paper we will show how we can use our theoretical results to argue why the asymptotic state of MHD turbulence is close to 0.5. The detailed shell-to-shell energy transfer also provide us important ideas for large-eddy simulations (see Debliquy et al. 6] for connection with large-eddy simulations) and EDQNM calculation, which assumes local energy transfers among wavenumber shells.

It is well known that compressible turbulence involves energy transfers from pressure fluctuations to the velocity and magnetic field 19, 20, 21, 22, 23]. The theoretical, numerical, and observational studies show that the energy spectrum deviates from Kolmogorov's spectrum. For example, Burgers equation, which represents fully compressible fluid, has energy spectrum proportional to $k^{-2}$. The theory of compressible turbulence is not yet developed as much as that for incompressible turbulence. Due to the uncertainty of energy spectrum and other properties in the inertial range, in this paper we have confined ourselves to the study of shell-to-shell energy transfer for incompressible turbulence only.

The outline of the paper is as follows: In Sec. 2 we compute the shell-to-shell energy transfer rates for nonhelical and helical MHD. In Sec. 3 we use our results to show why the asymptotic state of MHD turbulence has Alfvén ratio close to 0.5. The last section, Sec. 4, contains conclusions.

\section{CALCULATION OF THE SHELL-TO-SHELL ENERGY TRANSFERS}

In MHD turbulence, velocity ( $\mathbf{u})$ and magnetic fields $\mathbf{b}$ interact with each other and among itself to produce complex energy transfers. The energy exchange can take place between a $\mathbf{u}$ Fourier mode to $\mathbf{u}$ Fourier mode, between a $\mathbf{b}$ Fourier mode to $\mathbf{b}$ Fourier mode, or between $\mathbf{a} \mathbf{u}$ Fourier mode to $\mathbf{b}$ Fourier mode. These transfers are studied using Kraichnan's formula $S(\mathbf{k} \mid \mathbf{p}, \mathbf{q})$ [9] or mode-to-mode energy transfer rate $S(\mathbf{k} \mid \mathbf{p}, \mathbf{q})$. In this paper we use the mode-tomode energy transfer rates $\left(S^{Y X}\left(\mathbf{k}^{\prime}|\mathbf{p}| \mathbf{q}\right)\right)$ that represents the energy transfer rates from mode $\mathbf{p}$ of field $X$ to mode $\mathbf{k}$ of field $Y$, with mode $\mathbf{q}$ acting as a mediator [10,11]. Note that $\mathbf{k}^{\prime}+\mathbf{p}+\mathbf{q}=0$. This formalism is used for computing the shell-to-shell energy transfer because the earlier formalism suffers from ambiguity arising due to the third leg of the interaction (see [10, 11]). The mode-to-mode energy transfer rates in MHD turbulence are given by

$$
\begin{aligned}
S^{u u}\left(\mathbf{k}^{\prime}|\mathbf{p}| \mathbf{q}\right) & =-\Im\left(\left[\mathbf{k}^{\prime} \cdot \mathbf{u}(\mathbf{q})\right]\left[\mathbf{u}\left(\mathbf{k}^{\prime}\right) \cdot \mathbf{u}(\mathbf{p})\right]\right) \\
S^{b b}\left(\mathbf{k}^{\prime}|\mathbf{p}| \mathbf{q}\right) & =-\Im\left(\left[\mathbf{k}^{\prime} \cdot \mathbf{u}(\mathbf{q})\right]\left[\mathbf{b}\left(\mathbf{k}^{\prime}\right) \cdot \mathbf{b}(\mathbf{p})\right]\right), \\
S^{u b}\left(\mathbf{k}^{\prime}|\mathbf{p}| \mathbf{q}\right) & =\Im\left(\left[\mathbf{k}^{\prime} \cdot \mathbf{b}(\mathbf{q})\right]\left[\mathbf{u}\left(\mathbf{k}^{\prime}\right) \cdot \mathbf{b}(\mathbf{p})\right]\right), \\
S^{b u}\left(\mathbf{k}^{\prime}|\mathbf{p}| \mathbf{q}\right) & =\Im\left(\left[\mathbf{k}^{\prime} \cdot \mathbf{b}(\mathbf{q})\right]\left[\mathbf{b}\left(\mathbf{k}^{\prime}\right) \cdot \mathbf{u}(\mathbf{p})\right]\right),
\end{aligned}
$$

where the above four formulas denote the energy transfers from $\mathbf{u}(\mathbf{p})$ to $\mathbf{u}(\mathbf{k})$, from $\mathbf{b}(\mathbf{p})$ to $\mathbf{b}(\mathbf{k})$, from $\mathbf{b}(\mathbf{p})$ to $\mathbf{u}(\mathbf{k})$, and from $\mathbf{u}(\mathbf{p})$ to $\mathbf{b}(\mathbf{k})$ respectively. For the derivation the reader is referred to the original papers [10, 11].

Using the above formulas we compute the shell-to-shell energy transfer in MHD turbulence by summing up the energy transfer among the Fourier modes. The energy transfer rates from $m$-th shell of field $X(u$ or $b)$ to $n$-th shell of field $Y(u$ or $b)$ is

$$
T_{n m}^{Y X}=\sum_{\mathbf{k}^{\prime} \in n} \sum_{\mathbf{p} \in m}\left\langle S^{Y X}\left(\mathbf{k}^{\prime}|\mathbf{p}| \mathbf{q}\right)\right\rangle .
$$

The $\mathbf{p}$-sum is over $m$-th shell, and the $\mathbf{k}^{\prime}$-sum is over $n$-th shell $[9,11]$. Since $S^{Y X}\left(\mathbf{k}^{\prime}|\mathbf{p}| \mathbf{q}\right)$ is a fluctuating quantity, we perform ensemble average to compute the average shell-to-shell energy transfer. We compute the ensemble average of $S$ using a standard field-theoretic technique, a technique similar to EDQNM calculation [24, 25, 26]. The calculation is quite standard, and it can found in McComb [26] or Verma 11].

The function $\left\langle S^{Y X}\left(\mathbf{k}^{\prime}|\mathbf{p}| \mathbf{q}\right)\right\rangle$ depends on kinetic energy $\left(E^{u}(k)\right)$, magnetic energy $\left(E^{b}(k)\right)$, cross helicity $\left(H_{c}(k)\right)$, magnetic helicity $\left(H_{M}(k)\right)$, kinetic helicity $\left(H_{K}(k)\right)$, and mean magnetic field. The total cross helicity, magnetic helicity, and kinetic helicity are defined as $\mathbf{u} \cdot \mathbf{b} / 2, \mathbf{a} \cdot \mathbf{b} / \mathbf{2}$, and $\mathbf{u} \cdot \omega / 2$ respectively, where a and $\omega$ are vector potential and vorticity respectively. The spectrum of these quantities are defined appropriately (refer to Verma [1] for details). For simplification we compute $S$ for zero cross helicity and zero magnetic field, and in the inertial range using Kolmogorov's energy spectrum. To study the effects of kinetic and magnetic helicities, we have split $S$ into helical and 
nonhelical components. The simplified expression (given below) is a function of Alfvén ratio $\left(r_{A}=E^{b}(k) / E^{u}(k)\right)$, normalized magnetic helicity $\left(r_{M}=k H_{M}(k) / E^{b}(k)\right)$, and normalized kinetic helicity $\left(r_{K}=H_{K}(k) /\left(k E^{u}(k)\right)\right)$. Please note that we are working in three dimensions.

After lengthy algebra we obtain

$$
\frac{\left\langle S^{X Y}(v, w)\right\rangle}{\Pi}=\left[\frac{\left(K^{u}\right)^{3 / 2}(2 \pi)^{2}}{k^{6}}\right]\left[F_{\text {nonhelical }}^{X Y}(v, w)+F_{\text {helical }}^{X Y}(v, w)\right] .
$$

where $K^{u}$ is Kolmogorov's constant for MHD turbulence, and some of the $F_{\text {nonhelical }}^{Y X}$ and $F_{\text {helical }}^{Y X}$ are

$$
\begin{aligned}
F_{\text {nonhelical }}^{b b} & =\frac{\frac{1}{r_{A}} t_{4}(v, w)(v w)^{-11 / 3}+\frac{1}{r_{A}} t_{8}(v, w) w^{-11 / 3}+\frac{1}{r_{A}^{2}} t_{10}(v, w) v^{-11 / 3)}}{\eta^{*}\left(1+v^{2 / 3}\right)+\nu^{*} w^{2 / 3}}, \\
F_{\text {nonhelical }}^{u b} & =-\frac{\frac{1}{r_{A}^{2}} t_{2}(v, w)(v w)^{-11 / 3}+\frac{1}{r_{A}} t_{7}(v, w) w^{-11 / 3}+\frac{1}{r_{A}} t_{11}(v, w) v^{-11 / 3)}}{\eta^{*}\left(v^{2 / 3}+w^{2 / 3}\right)+\nu^{*}}, \\
F_{\text {nonhelical }}^{b u} & =-\frac{\frac{1}{r_{A}} t_{3}(v, w)(v w)^{-11 / 3}+\frac{1}{r_{A}^{2}} t_{6}(v, w) w^{-11 / 3}+\frac{1}{r_{A}} t_{12}(v, w) v^{-11 / 3)}}{\eta^{*}\left(1+w^{2 / 3}\right)+\nu^{*} v^{2 / 3}}, \\
F_{\text {helical }}^{b b} & =\frac{\frac{r_{M} r_{K}}{r_{A}} t_{4}^{\prime}(v, w)(v w)^{-11 / 3}+\frac{r_{M} r_{K}}{r_{A}} t_{8}^{\prime}(v, w) w^{-11 / 3}+\frac{r_{M} r_{M}}{r_{A}^{2}} t_{10}^{\prime}(v, w) v^{-11 / 3}}{\eta^{*}\left(1+v^{2 / 3}\right)+\nu^{*} w^{2 / 3}} .
\end{aligned}
$$

Here $\nu^{*}$ and $\eta^{*}$ are renormalized viscosity and resistivity parameters. In this paper, we consider (a) nonhelical MHD $\left(r_{M}=r_{K}=0\right.$ and different $\left.r_{A} \mathrm{~s}\right)$, and (b) helical MHD $\left(r_{A}=1, r_{K}=0.1, r_{M}=-0.1\right)$. For nonhelical MHD with $r_{A}=0.5,1,2$, the constants $\left(K^{u}, \nu^{*}, \eta^{*}\right)$ taken are $(0.55,2.1,0.5),(0.75,1.0,0.69),(1.0,0.64,0.77)$ respectively $($ see Verma [16, 27] for the procedure to compute these constants). For helical MHD, our choice of $r_{K}=0.1$ (small positive) and $r_{M}=-0.1$ (small negative) is one of the typical values taken in numerical simulations, or observed in astrophysical situations; for this case, the constants $K^{u}=0.78, \nu^{*}=1.0, \eta^{*}=0.69$ have been taken from Verma [28].

The wavenumbers shells are binned logarithmically with the $n$-th shell being $\left(k_{0} s^{n-1}, k_{0} s^{n}\right)$. Note that the parameter $s$ is similar to the scale-disparity parameter of Zhou [3]. We nondimensionalize the equations using the transformation [25]

$$
k=\frac{a}{u} ; \quad p=\frac{a}{u} v ; \quad q=\frac{a}{u} w
$$

where $a=k_{0} s^{n-1}$. The resulting equation is

$$
\frac{T_{n m}^{Y X}}{\Pi}=K_{u}^{3 / 2} \frac{1}{2} \int_{s^{-1}}^{1} \frac{d u}{u} \int_{u s^{m-n}}^{u s^{m-n+1}} d v \int_{|1-v|}^{1+v} d w(v w)\left[F_{\text {nonhelical }}^{Y X}(v, w)+F_{\text {helical }}^{Y X}(v, w)\right],
$$

which is independent of $a$ in the inertial range. The independence of $a$ implies self-similarity in the inertial range. From Eq. (7) we can draw the following inferences:

1. The shell-to-shell energy transfer rate is a function of $n-m$, that is, $\Phi_{n m}=\Phi_{(n-i)(m-i)}$. Hence, the turbulent energy transfer rates in the inertial range are all self-similar. Note that this property holds only in the inertial range.

2. $T_{n m}^{u b} / \Pi=-T_{m n}^{b u} / \Pi$, or $b$-to- $u$ energy transfer rates from shell $m$ to shell $n$ is equal and opposite to the $u$-to $b$ energy transfer rates from shell $n$ to shell $m$. Hence $T_{n m}^{b u} / \Pi$ can be obtained from $T_{m n}^{u b} / \Pi$ by inversion at the origin.

3. The MHD energy fluxes are related to the shell-to-shell energy transfers by the relationship

$$
\Pi_{Y>}^{X<}=\sum_{n=m+1}^{\infty}(n-m) T_{n m}^{Y X}
$$

4. Net energy gained by a $u$-shell from $u$-to- $u$ transfer is zero because of self similarity. However, a $u$-shell can gain or lose a net energy due to imbalance between $u$-to- $b$ and $b$-to- $u$ energy transfers. By definition, we can show that net energy gained by an inertial $u$-shell is

$$
\sum_{n}\left(T_{n m}^{u b}-T_{n m}^{b u}\right)+T_{n n}^{u b}
$$


Similarly, net energy gained by a $b$-shell from $b$-to- $b$ transfer is zero. However, net energy gained by an inertial $b$-shell due to $u$-to- $b$ and $b$-to- $u$ transfers is

$$
\sum_{n}\left(T_{n m}^{b u}-T_{n m}^{u b}\right)+T_{n n}^{b u}
$$

Now we compute the integrals of Eq. (7); we denote the nonhelical and helical parts by $\left(T_{n m}^{Y X}\right)_{\text {nonhelical }}$ and $\left(T_{n m}^{Y X}\right)_{\text {helical }}$ respectively. Their properties are described below.

\section{Nonhelical shell-to-shell energy transfer}

We compute the nonhelical shell-to-shell energy transfer using $F_{\text {nonhelical }}^{Y X}$. We have chosen $s=2^{1 / 32}$. This study has been done for various values of Alfvén ratios. Fig. 1 contains plots of $\left(T_{n m}^{Y X} / \Pi\right)_{n o n h e l i c a l}$ vs. $n-m$ for four typical values of $r_{A}=0.5,1,5,100$. The numbers on the plots represent energy transfer rates from shell $m$ to shells $m+1, m+2, \ldots$ in the right, and to shells $m-1, m-2, \ldots$ in the left. For $r_{A}=0.5$, the maxima of $b$-to- $u$ energy transfers $\left(T_{n m}^{u b}\right)_{n o n h e l i c a l} / \Pi$ and $\left(T_{n m}^{b u}\right)_{n o n h e l i c a l} / \Pi$ occurs at $m=n$, and its values are approximately \pm 0.1 respectively. The corresponding values for $r_{A}=5$ are approximately $\mp 0.053$. By observing the plots we find the following interesting patterns:

1. The $u$-to- $u$ energy transfer rate from shell $m$ to shell $n\left(T_{n m}^{u u}\right)_{n o n h e l i c a l} / \Pi$ is positive for $n>m$, and negative for $n<m$. Hence, a $u$-shell gains energy from smaller wavenumber $u$-shells, and loses energy to higher wavenumber $u$-shells, implying that the energy cascade is forward. Also, the absolute maximum occurs for $n=m \pm 1$, hence the energy transfer is local. For kinetic energy dominated regime, $s=2^{1 / 2}$ yields $T_{n m}^{u u} / \Pi \approx 35 \%$, similar to Kraichnan's Test Mean Field model (TFM) predictions [1].

2. The $b$-to- $b$ energy transfer rate $T_{n m}^{b b} / \Pi$ is positive for $n>m$, and negative for $n<m$, and maximum for $n=m \pm 1$. Hence magnetic to magnetic energy transfer is forward and local. This result is consistent with the forward magnetic-to-magnetic cascade $\left(\Pi_{b>}^{b<}>0\right)$ [11, 27].

3. For $r_{A}>1$ (kinetic energy dominated), kinetic to magnetic energy transfer rate $\left(T_{n m}^{b u}\right)_{n o n h e l i c a l} / \Pi$ is positive most of the shells. For $n-m<-30$ or so, the value is small and negative. These transfers have been illustrated in Fig. 3(a). Using Eq. (9) we find that each $u$-shell loses a net kinetic energy to $b$-shells, hence the turbulence is not steady. This phenomena is seen for all $r_{A}>1$, and it could be one of the processes responsible for dynamo action. For $s=2^{1 / 4},\left(T_{n n}^{b u}\right)_{\text {nonhelical }} / \Pi \approx 1.4$, and the $\left(T_{n m}^{b u}\right)_{\text {nonhelical }} / \Pi$ is positive for $n \geq m-1$ and negative otherwise.

4. For $r_{A}=0.5$ (magnetically dominated), magnetic to kinetic energy transfer rate $\left(T_{n m}^{u b}\right)_{\text {nonhelical }} / \Pi$ is positive for most of the shells (see Fig. (1). For $n-m<-30$ or so, the value is small and negative. In addition, using Eq. (8) we find that each $b$-shell loses a net magnetic energy to $u$-shells, hence the turbulence cannot be steady. This phenomena is seen for all $r_{A}<1$. For $s=2^{1 / 4},\left(T_{n m}^{u b}\right)_{n o n h e l i c a l} / \Pi$ is positive for $n \geq m-1$ and negative otherwise.

5. The observations of (3) and (4) indicate that kinetic to magnetic or the reverse energy transfer rate almost vanishes near $r_{A}=1$. We believe that the evolution of $M H D$ turbulence toward $r_{A} \approx 1$ in both decaying and steady-state is due to the above reasons. For $r_{A} \neq 1$, MHD turbulence is not steady. This result is similar to Pouquet et al.'s prediction of equipartition of kinetic and magnetic energy using EDQNM calculation 18|. An analogous result was discovered by Stribling and Matthaeus [29] in the context of the Absolute Equilibrium Ensemble (AEE) theory.

The steady-state value of $r_{A}$ in numerical simulations (e.g. Debliquy et al. [6], Dar et al. [10], Alexakis et al. [7], Mininni et al. [8], and Haugen et al. 30]) and solar wind (e.g., Matthaeus and Goldstein [31]) is around 0.5-0.6. The difference is probably because the realistic flows have more interactions than those discussed above, e.g., nonlocal coupling with forcing wavenumbers, coherent dissipative structures [32] etc.

6. When $r_{A}$ is not close to $1\left(r_{A} \leq 0.5\right.$ or $\left.r_{A}>5\right), u$-to- $b$ shell-to-shell transfer involves many neighbouring shells (see Fig. (1). This observation implies that $u-b$ energy transfer is somewhat nonlocal as predicted by Pouquet et al. [18].

7. We compute energy fluxes using $T_{n m}^{Y X}$, and find them to be the same as that computed by Verma [11, 27]. Hence both the results, flux and shell-to-shell energy transfer rates, are consistent with each other. 
Debliquy et al. [6] performed a decaying MHD turbulence simulations and computed the shell-to-shell energy transfer rates. The kinetic and magnetic helicity was approximately zero. Debliquy et al.'s results show that the shell-to-shell energy transfers are forward and local. They also find that in the magnetically dominated MHD, the energy transfer from the same shell is from magnetic field to velocity field. Since the numerical simulations start with $r_{A}=1$, the energy transfer rates for kinetic-energy dominated regime is not known numerically. Our theoretical results are in general agreement with the numerical results of Debliquy et al. As an example, the numerical values of shell-to-shell energy transfer rates shown in Fig. 9 of Debliquy et al. [6] $\left(r_{A} \approx 0.4\right)$ is similar to our theoretical results shown in Fig. 11. A major difference between theoretical and numerical values are for $T^{u b} / \Pi$ where theoretical value for $n=m$ is larger than its numerical counterpart.

Debliquy et al. [6] found that $T^{b u}$ changes sign near $r_{A} \approx 0.5$. In our theoretical calculation, the change of sign takes place around $r_{A} \approx 1$. These findings are very encouraging, and they yield explanation why the asymptotic state of Alfvén ratio is close to 0.5. The difference between theory and numerical simulations may be due to the neglect of large-scale non-Kolmogorov-like behaviour in theory, or due to the fact that Debliquy et al.'s results are based on decaying simulations, while the theoretical results assume steady-state. These issues need to be addressed.

Alexakis et al. [7] and Mininni et al. 8] computed shell-to-shell energy transfers in forced MHD. In the inertial range, the energy transfers are essentially local. However, the forcing velocity-shell (at large length-scale) provides energy to the large-scale magnetic field. The forcing velocity-shells also provide energy to the inertial range shells by nonlocal channel. Similar picture was observed by Dar et al. [10] in forced 2D MHD turbulence. Our theoretical results are consistent with the Alexakis et al.'s results in the inertial range. Unfortunately, the present theoretical calculation cannot predict the coupling with the forcing shell.

After the above discussion on nonhelical MHD, we move to helical MHD.

\section{Helical Contributions}

Now we present computation of $\left(T_{n m}^{Y X}\right)_{\text {helical }} / \Pi$, shell-to-shell energy transfer rates for helical MHD $\left(H_{M} \neq 0, H_{K} \neq\right.$ 0) [28]. To simplify the equation, we consider only nonAlfvénic fluctuations $\left(\sigma_{c}=0\right)$. We have chosen $r_{A}=1, r_{K}=$ $0.1, r_{M}=-0.1$. These values are one of the typical parameter values chosen in numerical simulations. We take $s=2^{1 / 4}$ to get a increased value for $\left(T_{n m}^{Y X}\right)_{\text {helical }} / \Pi$. For the above choice of parameters, Kolmogorov's constant $K^{u}=0.78$ [28]. In Fig. 2 we have plotted $\left(T_{n m}^{Y X}\right)_{\text {helical }} / \Pi$ vs $n-m$. Our results on helical shell-to-shell transfers are given below:

1. Comparison of Fig. 2 with Fig. 1 $\left(r_{A}=1\right)$ shows that helical energy transfers are order-of-magnitude lower than the nonhelical ones for the parameters chosen here $\left(r_{A}=1, r_{K}=0.1, r_{M}=-0.1\right)$. For maximal helicity, the helical and nonhelical values become comparable.

2. All the helical contributions are negative for $n>m$, and positive for $n<m$. Hence, helical transfers are from larger wavenumbers to smaller wavenumbers. This is consistent with the inverse cascade of energy due to helical contributions, as discussed by Pouquet et al. [18, 28].

3. We find that the helical shell-to-shell energy transfer rate $\left(T_{n m}^{u b}\right)_{\text {helical }}$ and $\left(T_{n m}^{b b}\right)_{\text {helical }}$ is significantly positive for $-50<n-m \leq 0$. This signals a nonlocal $b$-to- $b$ and $u$-to- $b$ inverse energy transfers. Hence, helicity induces nonlocal energy transfer between $b$-to- $b$ and $u$-to- $b$ wavenumber shells. This is in agreement with Pouquet et al.'s result [18] that "residual helicity" induces growth of large-scale magnetic field by nonlocal interactions.

The theoretical findings listed above are consistent with Pouquet's results based on EDQNM approximation [18] and flux calculations of Brandenburg et al. [33]. Alexakis et al. 7] and Mininni et al. [8] have computed shell-to-shell energy transfer in helical MHD turbulence; the helicity does change the energy transfer rates, however, in the absence of numerical value of normalized kinetic and magnetic helicity $\left(r_{K}, r_{M}\right)$, we are not able to compare our results with their numerical values.

In the next section we use our theoretical results and Debliquy et al.'s [6] numerical results to argue why the asymptotic state of MHD flows have $r_{A} \approx 0.4-0.6$.

\section{CONNECTION WITH MHD ASYMPTOTIC STATE $\left(r_{A} \approx 0.4-0.6\right)$}

The solar wind observations and numerical simulations show that the asymptotic state in the MHD flows have $r_{A} \approx 0.4-0.6$. We find in our theoretical analysis that for $r_{A}>1$, there is a preferential transfer of kinetic energy to magnetic energy; in fact, a $u$-shell loses a net amount of kinetic energy to $b$-shell. For $r_{A}<1$, the pattern of $u$-to- $b$ 
energy transfer is reversed, and there is a a net transfer of energy from magnetic to kinetic. This preferential energy transfer is minimum for $r_{A} \approx 1$. Our theoretical calculation however is based on an assumption of Kolmogorov's spectrum for the energy, which is not valid for the smaller wavenumber modes. Here we use Debliquy et al.'s [6] decaying simulations results for obtaining further insights into the large-scale energy transfers.

Debliquy et al.'s [6] showed that the energy flux from small-wavenumber $u$-sphere to small wavenumber $b$-sphere is positive for $r_{A}>0.63$, and becomes negative for lower $r_{A}$. The global (inclusive of all shells) $u$-to- $b$ energy transfer changes sign at $r=0.4$. Hence, the smaller wavenumbers shells also play an important role in energy transfers. As a result, the asymptotic state of MHD turbulence has Alfvén ration close to 0.5. Thus our detailed analysis of shell-to-shell energy transfer, and Debliquy et al.'s global and flux analysis is able to explain qualitatively why MHD turbulence evolves to asymptotic state with Alfvén ratio $r_{A} \approx 0.4-0.6$. Our theoretical predictions are consistent with the numerical results of Dar et al. [10] whose asymptotic Alfvén ratio $r_{A}$ is approximately 0.5. Alexakis et al. [7] and Mininni et al. 8] also find their asymptotic Alfvén ratio $r_{A}$ to be less than 1 .

\section{CONCLUSIONS}

In this paper we have computed the shell-to-shell energy transfers in MHD turbulence analytically. Our results provide theoretical explanation for the recently computed shell-to-shell energy transfers using direct numerical simulations. The contributions of nonhelical and helical terms have been calculated separately. We find that the nonhelical $u$-to- $u$ and $b$-to- $b$ shell-to-shell energy transfers are local as in fluid turbulence, i. e., most of energy from a wavenumber shell is transferred to the neighbouring shells. Comparatively, helical $u$-to- $b$ and $b$-to- $u$ energy transfers involves distant shells (nonlocal).

We find that the helical shell-to-shell energy transfer is backward, that is from larger wavenumbers to smaller wavenumbers. For $r_{K}=0.1, r_{M}=-0.1$, one of the typical values observed in numerical simulations, the helical shellto-shell energy transfer is order-of-magnitude smaller than the nonhelical ones. However, for maximal helicity, the helical shell-to-shell energy transfer is comparable to the nonhelical ones. In the present calculation, the parameters $r_{K}$ and $r_{M}$ have been chosen to be constants. This is a gross assumption considering that magnetic helicity and kinetic helicity have different signs at different scales. Even then we obtain results which are consistent with recent numerical results and earlier theories (Frisch et al. [34]). Hence, the present calculation appears to capture some of the essential features of helical MHD turbulence.

Our results show that the inertial-range shell-to-shell for $r_{A}>1$, there is a preferential transfer of kinetic energy to magnetic energy; the direction of energy transfer switches for $r_{A}<1$. Debliquy et al.'s [6] numerical simulations provide us important clues for the energy transfers at smaller wavenumbers. Using these results we can argue why the asymptotic state of MHD turbulence evolves to Alfvén ratio of 0.5.

Our theoretical results are in general agreement with the numerical results of Debliquy et al. [6], Dar et al. [10], Alexakis et al. [7] and Mininni et al. [8], who observe local energy transfer in the inertial range. In forced MHD turbulence with large-scale velocity forcing, a significant energy transfer to large-scale magnetic field and nonlocal energy transfer to inertial-range magnetic field were found. Since the nonlinear energy transfers involves only the velocity and magnetic field variables, we expect that the features of energy exchange in the inertial range should remain approximately the same in decaying and forced MHD. However, the forcing at large-scale velocity shell would affect the large-scale magnetic field, and could also induce nonlocal interations. A theoretical model with forcing will be useful to understand forced MHD turbulence.

Detailed pictures of energy transfer studied here is useful for understanding various physical process, for example, dynamo mechanism to generate magnetic field in astrophysical objects. These results are also useful in modelling MHD flows and in simulations. For example, we need to model backscatter and forward energy transfer for large-eddy simulations. Refer to Debliquy et al. [6] for discussion on backscatter and forward energy transfer in Fourier as well as real space.

In EDQNM analysis, the wavenumber shells are logarithmically binned. In the present analysis of MHD turbulence, and many papers on fluid turbulence show local energy transfers among wavenumber shells for nonhelical MHD. Hence, the local energy transfer assumptions made in EDQNM analysis is valid at least for nonlocal MHD [35]. The energy transfers are somewhat more complex for helical MHD.

Pouquet et al. 18 performed extensive EDQNM analysis of MHD turbulence and showed that both local and nonlocal interactions exist in MHD turbulence. The local interactions cause the energy cascade, while the nonlocal ones cause equipartition of kinetic and magnetic energies. Pouquet et al. argued for inverse cascade of magnetic energy from the competition between helicity and Alfvén effect. Our analytic calculation also predicts inverse magnetic-energy cascade due to helicity. Our calculation shows that near equipartition of magnetic and kinetic energy is due to complex process involving inertial-range shell-to-shell interactions and small wavenumber shells. This picture is some what different than that of Pouquet et al. 
To conclude, the shell-to-shell energy transfer rates provide important insights into inertial-range energy exchange processes in MHD turbulence.

\section{Acknowledgments}

MKV gratefully acknowledges useful comments and suggestions by Diego Donzis, Daniele Carati, and Olivier Debliquy. Part of the work was supported by a project from Department of Science and Technology, India.

[1] R. H. Kraichnan, J. Fluid Mech. 47, 525 (1971).

[2] J. A. Domaradzki and R. S. Rogallo, Phys. Fluids A 2, 413 (1990).

[3] Y. Zhou, Phys. Fluids 5, 1092 (1993).

[4] Y. Zhou and C. G. Speziale, Appl. Mech. Rev. 51, 267 (1998).

[5] M. K. Verma, A. Ayyer, O. Debliquy, S. Kumar, and A. V. Chandra, Pramana 65, 297 (2005).

[6] O. Debliquy, M. K. Verma, and D. Carati, Phys. Plasmas 12, 42309 (2005).

[7] A. Alexakis, P. D. Mininni, and A. Pouquet, ArXiv physics/0505183

[8] P. D. Mininni, A. Alexakis, and A. Pouquet, ArXiv physics/0505189

[9] M. Lesieur, Turbulence in Fluids - Stochastic and Numerical Modelling (Kluwer Academic Publishers, Dordrecht, 1990).

[10] G. Dar, M. K. Verma, and V. Eswaran, Physica D 157, 207 (2001).

[11] M. K. Verma, Phys. Rep. 41, 229 (2004).

[12] M. K. Verma, Phys. Plasmas 6, 1455 (1999).

[13] S. Sridhar and P. Goldreich, Astrophys. J. 432, 612 (1994).

[14] P. Goldreich and S. Sridhar, Astrophys. J. 438, 763 (1995).

[15] M. K. Verma, Phys. Rev. E 64, 26305 (2001).

[16] M. K. Verma, Phys. Plasmas 8, 3945 (2001).

[17] M. Hnatich, J. Honkonen, and M. Jurcisin, Phys. Rev. E 64, 56411 (2001).

[18] A. Pouquet, U. Frisch, and J. Léorat, J. Fluid Mech. 77, 321 (1976).

[19] L. D. Landau and E. M. Lifsitz, Fluid Mechanics (Pergamon Press, Oxford, 1987).

[20] G. P. Zank and W. H. Matthaeus, Phys. Fluids A 3, 69 (1991).

[21] G. P. Zank and W. H. Matthaeus, Phys. Rev. Lett. 64, 1243 (1990).

[22] J. Cho and A. Lazarian, Mon. Not. R. Astron. Soc. 345, 325 (2003).

[23] M. K. Verma and G. Dar, in Nonlinear Dynamics and Computational Physics, edited by V. Sheorey (Narosa, New Delhi, 1998), p. 61.

[24] R. H. Kraichnan, Phys. Fluids 8, 1385 (1965).

[25] D. C. Leslie, Development in the Theory of Turbulence (Oxford University Press, Claredon, 1973).

[26] W. D. McComb, Physics of Fluid Turbulence, The (Oxford University Press, Claredon, 1990).

[27] M. K. Verma, Pramana 61, 577 (2003).

[28] M. K. Verma, Pramana 61, 707 (2003).

[29] T. Stribling and W. H. Matthaeus, Phys. Fluids B 2, 1979 (1990).

[30] N. E. L. Haugen, A. Brandenburg, and W. Dobler, Astrophys. J. 597, L141 (2003).

[31] W. H. Matthaeus and M. L. Goldstein, J. Geophys. Res. 87, 6011 (1982).

[32] W. H. Matthaeus and G. Lamkin, Phys. Fluids 29, 2513 (1986).

[33] A. Brandenburg, Astrophys. J. 550, 824 (2001).

[34] U. Frisch, A. Pouquet, J. Léorat, and A. Mazure, J. Fluid Mech. 68, 769 (1975).

[35] Y. Zhou, W. H. Matthaeus, and P. Dmitruk, Rev. Mod. Phys. 76, 1015 (2005).

\section{Figure Captions}

Figure 1: The plots of shell-to-shell energy transfers $\left(T_{n m}^{Y X}\right)_{n o n h e l i c a l} / \Pi$ vs. $n-m$ for zero helicities $\left(\sigma_{c}=\right.$ $\left.r_{K}=r_{M}=0\right)$ and Alfvén ratios $r_{A}=0.5,1,4,100$. Here $s=2^{1 / 32}$. The $u$-to- $u, b$-to- $u, u$-to- $b$, and $b$-to- $b$ are represented by dotted, dashed, chained, and solid lines respectively. For $r_{A}=0.5$, the maxima of $\left(T_{n m}^{u b}\right)_{n o n h e l i c a l} / \Pi$ and $\left(T_{n m}^{b u}\right)_{n o n h e l i c a l} / \Pi$ are \pm 0.1 respectively, out of scale of the plot. The corresponding values for $r_{A}=5$ are $\mp 0.053$.

Figure 2: Helical contributions to shell-to-shell energy $\operatorname{transfer} s\left(T_{n m}^{Y X}\right)_{\text {helical }} / \Pi$ vs. $n-m$ in helical MHD with $r_{A}=1, r_{K}=0.1, r_{M}=-0.1$ and $\sigma_{c}=0$. Here $s=2^{1 / 4}$. 
Figure 3: Schematic illustration of nonhelical shell-to-shell energy transfers $T_{n m}^{Y X} / \Pi$ in the inertial range for (a) kinetic-energy dominated regime, and (b) magnetic-energy dominated regime. In (a) the $u$-to- $b$ energy transfer rate $T_{n m}^{b u} / \Pi$ is positive for $n \geq m-1$, and negative otherwise, while in (b) the $b$-to- $u$ energy transfer rate $T_{n m}^{u b} / \Pi$ is positive for $n \geq m-1$, and negative otherwise. The $u$-to- $u$ energy transfer rate $T_{n m}^{u u}$, and the $b$-to- $b$ energy transfer rate $T_{n m}^{b b}$ are forward and local. 

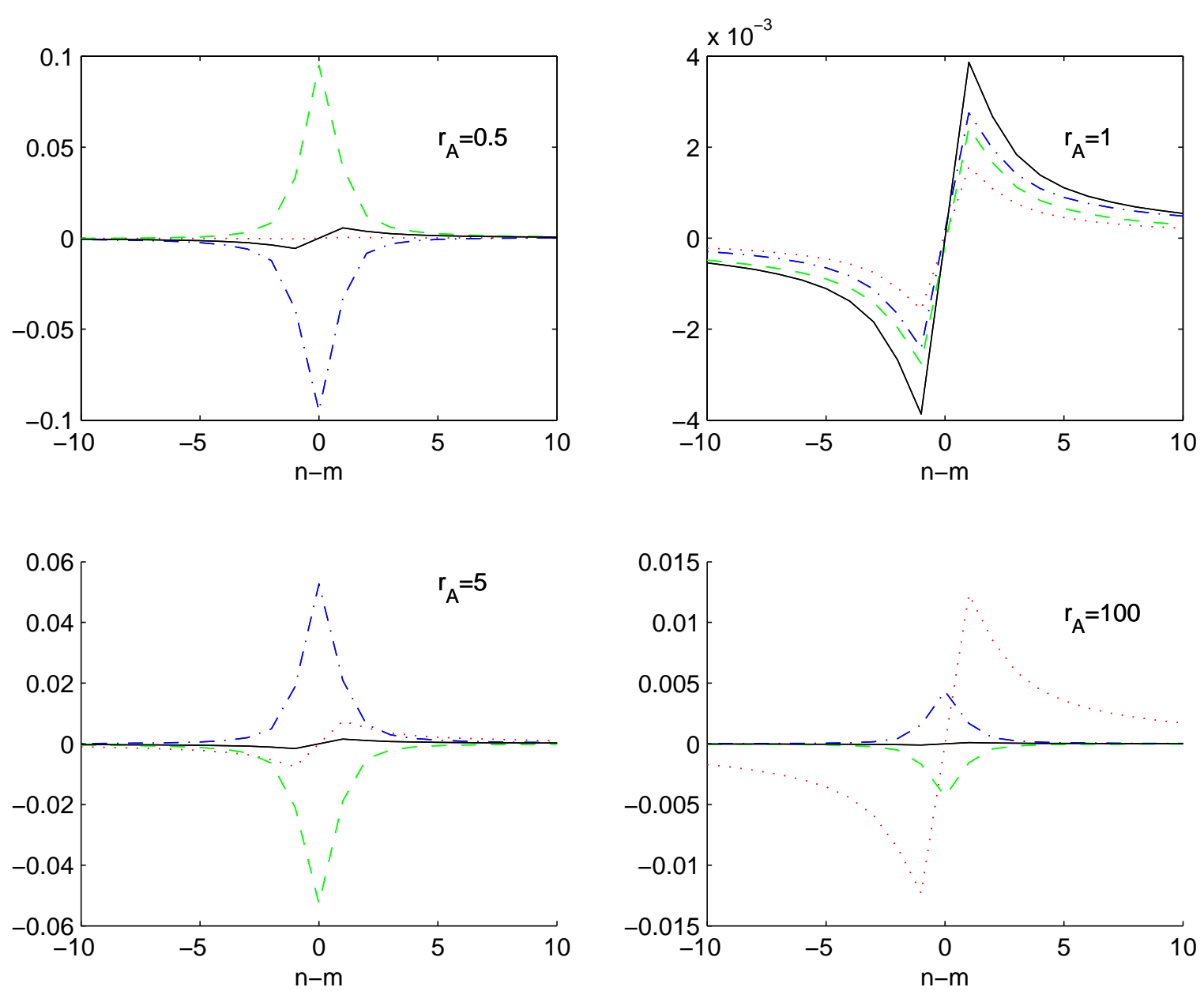

Figure 1: The plots of shell-to-shell energy transfers $\left(T_{n m}^{Y X}\right)_{n o n h e l i c a l} / \Pi$ vs. $n-m$ for zero helicities $\left(\sigma_{c}=r_{K}=r_{M}=0\right)$ and Alfvén ratios $r_{A}=0.5,1,4,100$. Here $s=2^{1 / 32}$. The $u$-to- $u, b$-to- $u, u$-to- $b$, and $b$-to- $b$ are represented by dotted, dashed, chained, and solid lines respectively. For $r_{A}=0.5$, the maxima of $\left(T_{n m}^{u b}\right)_{n o n h e l i c a l} / \Pi$ and $\left(T_{n m}^{b u}\right)_{n o n h e l i c a l} / \Pi$ are \pm 0.1 respectively, out of scale of the plot. The corresponding values for $r_{A}=5$ are $\mp 0.053$.

\section{FIGURES}




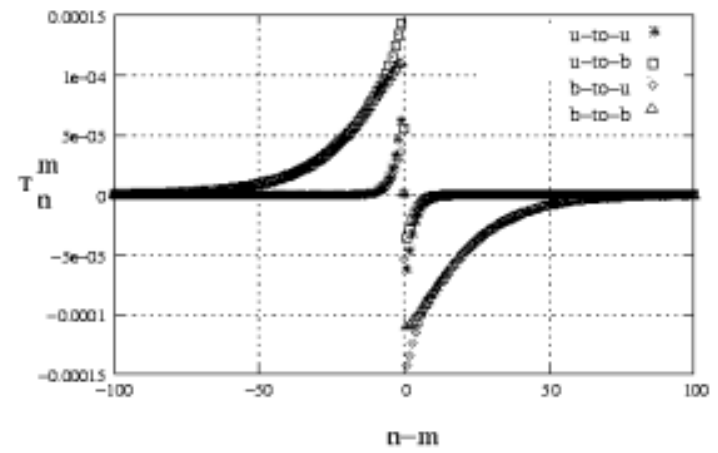

Figure 2: Helical contributions to shell-to-shell energy $\operatorname{transfer} s\left(T_{n m}^{Y X}\right)_{h e l i c a l} / \Pi$ vs. $n-m$ in helical MHD with $r_{A}=1, r_{K}=$ $0.1, r_{M}=-0.1$ and $\sigma_{c}=0$. Here $s=2^{1 / 4}$.

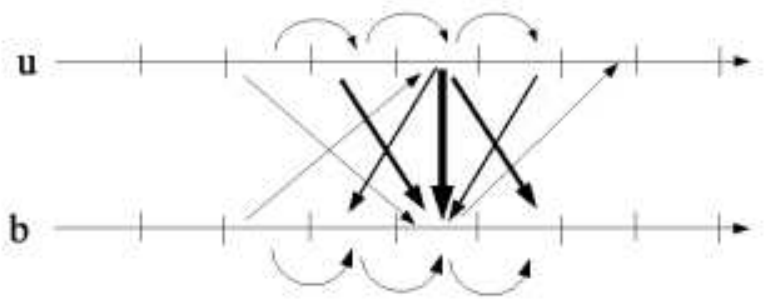

(a)

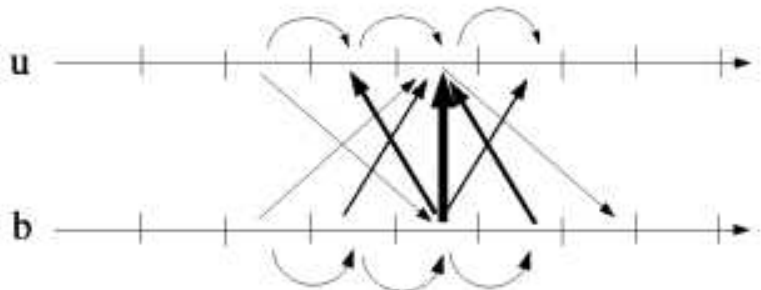

(b)

Figure 3: Schematic illustration of nonhelical shell-to-shell energy transfers $T_{n m}^{Y X} / \Pi$ in the inertial range for (a) kinetic-energy dominated regime, and (b) magnetic-energy dominated regime. In (a) the $u$-to- $b$ energy transfer rate $T_{n m}^{b u} / \Pi$ is positive for most $n$, while in (b) the $b$-to- $u$ energy transfer rate $T_{n m}^{u b} / \Pi$ is positive for most $n$. The $u$-to- $u$ energy transfer rate $T_{n m}^{u u}$, and the $b$-to- $b$ energy transfer rate $T_{n m}^{b b}$ are forward and local. 